\title{
Der ,Ekel' in der Erzählung Mit Messer und Gabel von Doris Dörrie $^{1}$
}

\author{
Tijen Köşetaş (D), İstanbul
}

https://doi.org/10.37583/diyalog. 1030742

\section{Abstract (Deutsch)}

Ekel wird im breiten Sinne als eine Abwehrreaktion, eine Ablehnung definiert. Das Ekelgefühl ist jedem Menschen angeboren und Disziplinen, wie die Psychologie und die Anthropologie, einigen sich darüber, dass Ekel ein menschliches Gefühl ist. Obwohl Ekel eine bedeutende Komponente des Gefühlslebens darstellt, ist die Forschung in diesem Bereich äußerst gering.

Die vorliegende Arbeit beschäftigt sich mit den Gründen sowie den Folgen des Ekels auf Grundlage der Erzählung Mit Messer und Gabel von Doris Dörrie. In komprimierter Form thematisiert der Text die vier Sinne - Geräusch, Geruch, Tastsinn und Sehsinn -, die mit Ekel verbunden sind, und stellt deren Auswirkung dar. Im Hintergrund dieser Überlegungen beschäftigt sich die vorliegende Arbeit mit folgenden Fragen: Wie wird Ekel von Doris Dörrie dargestellt? Wie versucht die Protagonistin der Erzählung das Ekelempfinden zu bewältigen? Was sind weitere Gefühlszustande, die der Ekel bei der Protagonistin evoziert?

Methodisch lehnt sich die Arbeit an die Phänomenologie an. In seinem Werk Ekel. Hochmut, Haß. Zur Phänomenologie feindlicher Gefühle beschäftigt sich Aurel Kolnai ausschließlich mit Ekel und vergleicht diesen mit anderen feindlichen Gefühlen. Für eine ausführliche Analyse der Erzählung sollen Kolnais Überlegungen und Differenzierungen $\mathrm{zu}$ Ekel und anderen antagonistischen Gefühle herangezogen werden.

Schlüsselwörter: Ekel, Ekelgefühl, Basisemotionen, Angst, Phänomenologie.

\section{Abstract (English)}

\section{The Motif of Disgust in the Short Story Mit Messer und Gabel by the Author Doris Dörrie}

Disgust is classified as one of the basic emotions, along with joy, happiness, jealousy and dread. There are many definitions of disgust. However, it is basically considered to be a strong response, towards something that is felt to be unpleasant. Although it is considered to be one of the basic emotions, not many literary works or movies choose to put this dark emotion at the center of their plot. In that regard, is the story Mit Messer und Gabel one of the very few literary works that basically deals with disgust on many different levels.

This paper specifically concentrates on the causes and the consequences of disgust in the story Mit Messer und Gabel. Furthermore, it aims to answer the following questions: How is disgust being

1 Dieser Aufsatz wurde auf dem XV. Internationalen Türkischen Germanistenkongress, der vom 30. September-02. Oktober in Edirne stattfand, präsentiert. Die vorliegende Arbeit ist eine erweiterte Version des Vortrags. 
depicted by the Author Doris Dörrie? How does the Protagonist try to fight this emotion and what other emotions are being evoked due to disgust?

The method applied in this paper is based upon the thoughts of the phenomenologist Aurel Kolnai. In his work Ekel, Hochmut, Haß. Zur Phänomenologie feindlicher Gefühle, deals Kolnai exclusively with disgust as a basic emotion and compares it with other hostile feelings.

Keywords: Disgust, Feeling of Disgust, Basic Emotions, Fear, Phenomenology. 


\section{EXTENDED ABSTRACT}

Disgust is classified as one of the basic emotions along with joy, happiness, jealousy and dread. Disciplines like anthropology and psychology define it as one of the key feelings of human beings, as well. There are many definitions of disgust. However, it is basically considered to be a strong response, towards something that is felt to be unpleasant. Although it is considered to be one of the basic emotions, not many literary works or movies choose to put this dark emotion at the center of their plot. In that regard, is the story Mit Messer und Gabel by the Author Doris Dörrie one of the very few literary works that basically deals with disgust on many different levels.

This paper specifically concentrates on the causes and the consequences of disgust in the story Mit Messer und Gabel. Furthermore, it aims to answer the following questions: How is disgust being depicted by the Author Doris Dörrie? How does the protagonist try to fight this emotion and what other emotions are being evoked due to disgust?

The method applied in this paper is based upon the thoughts of the phenomenologist Aurel Kolnai. In his work Ekel, Hochmut, Haß. Zur Phänomenologie feindlicher Gefühle, Kolnai exclusively deals with disgust as a basic emotion and compares it with other hostile feelings.

Dörrie places a nameless protagonist at the center of her story, who happens to be sensitive towards certain sounds, smells, and the look of certain foods. Whenever she is confronted with these certain things, she is overwhelmed by a strong feeling of disgust. Sounds like eating an apple or the sound of slippers rubbing against each other create a kind of discomfort in her. Due to this uneasiness and distress, her body starts showing physical reactions she is not able to control. Since she does not know how to cope with this feeling, she thinks that leaving the place or the object that causes this feeling, is the best solution. She does not think about communicating her problem or opening up to her parents because there is a certain distance between them.

The protagonist believes in the power of love. She thinks that disgust can only be felt when there is no love. When she later falls in love and gets married, she believes the problem to be resolved. The relationship that seems to be harmonious at the beginning, takes a dramatic turn, when her husband decides to come home for lunch. The protagonist observes that her husband has an individual way of preparing and eating food. He puts different kinds of food on the plate and eventually mixes them with his fork. She is not only disgusted by the sight of this mixture, but also by the sound made by the fork while mixing everything together. In order to avoid this, she decides to cook for her husband. She prepares solid foods like salad and steak and avoids mushy foods, and foods that are sticky and soft. The protagonist likes to cook and prepare things in a certain way. Furthermore, she attaches a lot of importance to visual presentation. The sight of mixed food that do not belong together, causes her to feel disgust. Disappointed with the food served by his wife, the husband decides to cook for himself again. Thus, she unwillingly has to leave the kitchen. She also distances herself from her husband and tries to avoid him in every possible way.

The husband who cannot understand the drastic change in her attitude is confused. He wants to talk with her and is expecting an explanation. He locks the kitchen door and wants her to take a seat. He is preparing lunch while they are talking. The protagonist is feeling an increasing discomfort. She loses complete control, the moment he starts mixing the food on his plate. 
The feeling of disgust makes her life so unbearable that she commits a crime and kills her husband. That she was locked in the kitchen made her feel trapped and terrified. Disgust as the dominating force in her life, caused her to be miserable und ruined her relationships.

Obwohl Ekel eine bedeutende Komponente des Gefühlslebens darstellt, ist er bis dato als äußerst gering untersuchtes Thema anzusehen. In seinem Werk Ekel (2002) beschäftigt sich Winfried Menninghaus (1952) mit den grundlegenden ,theoretische[n] Beschreibungen [...] Verwendungen des Ekels"(Menninghaus 2002: 13) und benennt etliche Gründe dafür, weshalb diese Emotion, obwohl sie genau wie Angst, Ärger, Neid und Freude zu den Basisemotionen gehört, die jedem Menschen angeboren sind, im engeren Sinne behandelt wird. Menninghaus unterstreicht die Schwierigkeit, eine umfassende Geschichte des Ekels zu verfassen. Einer der Gründe soll der Mangel an Quellen in den Archiven sein. Des Weiteren sollen die Ausprägungen dieser ,als unwürdig, undezent" (ebd.: 9) definierten Emotion ebenfalls, sehr ungern aufgezeichnet, registriert und übertragen worden sein. Dies bildet somit den Grund einer weiteren Lücke in diesem Bereich. Demzufolge erweist sich Ekel als ein verdecktes Thema, dessen Gewicht als Basisemotion für Jahrzehnte übersehen worden ist. Doch Menninghaus' Werk rückt diese Basisemotion ins Blickfeld und leistet dabei einen wichtigen Beitrag, in dem es sich mit den „Theoretisierungen des Ekels“ (vgl. ebd.) auseinandersetzt und Licht auf die Rolle des Ekels in der Ästhetik sowie in der Kunst in den vergangenen 250 Jahren wirft. Es finden sich unterschiedliche Definitionen zu Ekel. Menninghaus definiert diese Emotion als ,eine der heftigsten Affektionen des menschlichen Wahrnehmungssystems“ (ebd.: 7).

In seinem Werk The Expression of the Emotions in Man and Animals (1872) beschreibt Charles Darwin (1809-1882) Ekel als „something offensive to the taste“ (Darwin 1872). William Ian Miller (1946) demgegenüber beschreibt Ekel in seinem Werk The Anatomy of Disgust (1997) mit den Worten, "Disgust is a feeling about something and in response to something [...]" (Miller 1997: 8). Damit deutet Miller darauf hin, dass Ekel eine Art Reaktion darstellt, die nicht ohne Grund auftaucht und die sich auf etwas Bestimmtes richtet.

Vor dem Hintergrund dieser Überlegungen beschäftigt sich die vorliegende Arbeit mit dem Motiv des Ekels anhand der Erzählung Mit Messer und Gabel (1989) von Doris Dörrie (1955). Daraus resultieren folgende Fragen, denen hier nachgegangen wird: Wie wird Ekel in der Erzählung dargestellt? Wie geht die Hauptfigur der Erzählung mit der Ekelempfindung um? Und was sind ihre Schutzmechanismen, die sie gegen diese Emotion einführt?

Für eine genauere Analyse werden im Folgenden insbesondere die Überlegungen des Phänomenologen Aurel Kolnais (1900-1973) herangezogen. Aurel Kolnai ist ein deutschsprachiger Philosoph jüdischer Herkunft und gehört $\mathrm{zu}$ den bedeutendsten Denkern des 20. Jahrhunderts. Kolnais Essay Der Ekel ist 1929 veröffentlicht worden. Dieser knapp 50 Seiten langer Essay, ist laut Menninghaus, ,immer noch das Fundament einer jeden Beschreibung des Ekels“ (Menninghaus 2002: 28). Um ein genaueres Bild über den Ekel zu vermitteln, vergleicht Kolnai den Ekel mit anderen Zuständen, wie der „Angst“, dem „Mißfallen“, und dem „Haß“ (Kolnai 2007: 8). Da 
diese „Ablehnungstönungen“ (ebd.), auch in Doris Dörries Text thematisiert werden, erweist sich Kolnais Werk für diese Untersuchung als relevant. Mit den Worten „Das Ekelhafte grinst, starrt, stinkt uns ,an“" (ebd.: 17) beschreibt Kolnai den Ekel in seinem Werk Ekel, Hochmut, Haß (2007). Damit akzentuiert er die Bösartigkeit, die Schamlosigkeit und den provokativen Charakter des Ekelhaften. Hinzukommt, dass der Ekel etwas Heimtückisches an sich hat, da er unerwartet auftaucht.

Doch im breiten Sinne beschreibt Kolnai den Ekel als eine „Abwehrreaktion“ (ebd.: 8) und verschafft mit seinem Text einen tiefen Einblick in diese Emotion. Tiefverankerte Vorurteile, zumeist kulturelle Differenzen, können zur Distanzierung und Abwehr führen, da sie mit dem eigenen Vertrauten nicht übereinstimmen. Das Essverhalten und die Küche anderer Kulturen würde als Beispiel und Grund für die Abwehr dienen. Im Übrigen sollen auch die „Klebrigen, Feuchten, Lauwarmen“ (Kolnai 2007: 9) sowie die „Schwabbligen, Schleimigen und Breiigen“ (ebd.: 27) Gegenstände, die mit dem Tastsinn verbunden sind, als ekelerregend empfunden werden.

In ihrer Erzählung Mit Messer und Gabel (1989) setzt Doris Dörrie eine namenlose Ich-Erzählerin in den Mittelpunkt. Der Text wird in alltäglicher Sprache und rückwärts erzählt. Dörries' kurzer, jedoch inhaltlich dichter Text thematisiert in komprimierter Form die vier Sinne - Gehör-, Geruch-, Tast- und Sehsinn - und deren Relation zu Ekel. Doch hauptsächlich sind es im Text das Gehör und der Sehsinn, die als Hauptträger des Ekels fungieren und hervorgehoben werden.

Der Text erzählt von einer Protagonistin, die vom Ekel befangen ist. Darüber hinaus werden unterschiedliche Formen des Empfindens von Ekel behandelt. Der Ekel bezieht sich hier nicht eindeutig auf einen bestimmten Gegenstand, wie etwa Nahrung oder Sexualität, wie es im Film Repulsion (1965) von Roman Polanski (1933) der Fall ist, sondern differenziert ausgehend von der Lage des Protagonisten Roquentin in JeanPaul Sartres“ (1905-1980) Roman Der Ekel (1938), wo dieser sich auf die eigene sowie die sinnlose menschliche Existenz gerichtet ist. Ekel bezieht sich bei der Protagonistin auf diverse Zustände und Dinge, was ihre Verortung des eigenen Ekelempfindens erschwert.

Bereits am Anfang der Erzählung äußert sie, dass die ersten Ekelempfindungen bereits durch ihre Eltern ausgelöst wurden. In den folgenden zwei Zitaten werden ihre seelischen und körperlichen Vorgänge genau dargestellt. Ihre Konfrontation mit dem Ekelgefühl drückt sie folgend aus:

Wenn meine Mutter einen Apfel ißt, macht sie so komische Geräusche, da läuft es mir eiskalt den Rücken runter, ich fange an zu zittern und würde sie am liebsten umbringen. Das war schon immer so, früher bin ich einfach aus dem Zimmer gegangen. [...] hätte ich ihr sagen sollen, daß es mich anekelt, wie sie einen Apfel ißt? (Dörrie 1990: 111)

Mein Vater hat immer seine Füße aneinander gerieben. [...], so sehr ich mich auch bemüht habe, wegzuhören, immer auf dieses leise, schabende Geräusch von seinen Hausschuhen horchen müssen. Hat mich ganz verrückt gemacht, manchmal mußte ich mir die Ohren zuhalten, um ihn nicht anzuschreien. (vgl. ebd.) 
Laut Kolnai hat „Alles Ekelhafte [...] etwas [...] Auffallendes [...]“ (Kolnai 2007: 23) an sich. In diesen vorstehend zitierten Fällen werden die auffälligen Verhaltensweisen der Eltern hervorgehoben. Die zwei unterschiedlichen Geräusche, wie das Essen eines Apfels durch die Mutter und das Aneinander-Reiben der Hausschuhe des Vaters, dienen als Auslöser für den erlebten Ekel der Protagonistin. Beides wird von ihr als aufdringlich empfunden. Der Grad ihrer Betroffenheit ist dermaßen hoch, dass ihr das Umbringen der Mutter oder das Anschreien des Vaters als eine endgültige Lösung erscheint. Dieses Empfinden versucht sie auf unterschiedliche Weise zu zügeln, ja sogar zu verdrängen. Da diese Geräusche wenig Gemeinsamkeit aufweisen, ist darauf hinzuweisen, dass ihre Ekelempfindung sich auf keine ausschließliche Form bezieht. Als Hauptträger des Ekels nennt Kolnai den ,[...] Geruchs-, [...] Gesichts- und [...] Tastsinn“ (vgl. ebd.: 24). Er räumt jedoch dem Gehör- und Sehekel ebenfalls einen breiten Raum ein und erklärt, wie die letzteren zwei sich von den übrigen Sinnen unterscheiden. „Töne und Geräusche ,verraten“ nur Gegenstände und ,präsentieren ' sie nicht in dem Sinne, wie es Gesichts-, Tast-, Geruchsempfindung“ (ebd.: 25) vermögen. ,[...] Ekel [...] kann durch das Gehör im allgemeinen überhaupt nicht vermittelt werden [...].“ (vgl. ebd.). Kolnai interpretiert das Gehör als eine schwächere Art der Rezeption der Ekelempfindung. Er akzentuiert und betrachtet den Ausgangspunkt des Gehörekels als Assoziation. So wird der Ekel durch die Assoziation des Geräusches mit einem gewissen Gegenstand hervorgerufen. Doch im Vergleich zu den Hauptträgern des Ekels soll er eine weitaus geringere Wirkung erzeugen, was jedoch im Falle der Protagonistin nicht zutrifft. Obwohl sie selbst keine Begründung für ihre Störung preisgibt, ist es laut ihrer körperlichen Reaktionen und ihrer Äußerungen evident, dass sie diese Geräusche mit etwas Tieferem und Unangenehmen assoziiert und dass diese sie in ein Unbehagen versetzen. Um sich ihres Problems zu entledigen, sucht sie vergebens nach Schutzmechanismen. Doch die Ablehnung des Zustands durch das Verlassen des Umfeldes erscheint für sie als einzige gangbare Lösung.

Ein wesentlicher Grund für den Ekel und ihre Reaktion ist, dass diese Geräusche von ihren Eltern stammen. Kolnai betont, dass , ,...] in der Ekelintention eine gewisse Geringschätzung ihres Objekts, ein Gefühl der Überlegenheit enthalten“ (Kolnai 2007: 18) ist. Wenn die Protagonistin sich über das Handeln ihrer Eltern äußert, sind ihre Aussagen mit Animosität, Hass und Abneigung gefärbt. Sie hat nicht das Bedürfnis, sich über ihr Ekelempfinden ihren Eltern gegenüber zu äußern. Das Nicht-KommunizierenWollen mit den Eltern deutet auf eine dysfunktionale Beziehung und eine zusätzliche Geringschätzung hin. Die Eltern werden als störend empfunden und deswegen soll auch der Kontakt zu ihnen auf minimaler Ebene gehalten werden.

Geräusche sind nicht das Einzige, die bei ihr Ekel auslösen. Intensiver Geruch von Brot oder das Berühren der fettigen Haare ihres Freundes führen bei ihr ebenfalls zu Ekelgefühlen. „Ich wusch sie ihm jeden Tag, und trotzdem waren sie fettig. Irgendwann haben sie mich an alte Spaghetti erinnert. Ich konnte ihm nicht mehr über den Kopf streicheln." (Dörrie 1990: 112)

Sie empfindet die Haare ihres Freundes als kontaminierend, und wenn sie sie berührt, wird sie von einem unguten Gefühl überwältigt. Deshalb möchte sie sich nicht 
mehr in seiner Nähe befinden und trifft den Entschluss, sich von ihm endgültig zu trennen.

Kolnai bezieht sich in seinem Werk auf zwei unterschiedliche Gegenstände, die beide als ekelhaft gelten, doch sich von der Eigenschaft her unterscheiden.

Es gibt vom praktisch-funktionalen Standpunkt aus zweierlei ekelhafte Gegenstände: solche, die sozusagen schon von Natur wegen ekelhaft sind, und solche, die es nur unter ganz bestimmten Umständen werden. Zur ersten Gruppe gehören namentlich die Exkremente und die schlechthin fauligen Stoffe. [...] Das Ekelgefühl gegen sie ist etwas „Natürliches“. [...], „ekelhaft ist hier ein Charakteristikum [...]. Anders verhält es sich mit Gegenständen, die irgendwie noch ,funktionsfähig“ sind, im Leben stehen: Speisen, Tiere, Lebensgebilde. Hier ist das Ekelgefühl stets - sei es auch wie allgemein immer „mutwilliger“, mehr auf einer „Entscheidung“ des Subjekts zugunsten der Abwehr beruhend. [...] Die zweite Unterscheidung betrifft den im verabscheuten Gegenstande fallweise wohnenden „Anspruch“. Gewisse unter ihnen sind uns gegenüber vollkommen passiv; solange wir sie nicht ,aufsuchen“ [...]. Oft aber liegt eine Aggression, eine aufgezwungene - etwa dauernde - aktuelle Nähe vor, [...]: eine aufgenötigte Beziehung, ein Wertanspruch des Gegenstandes, ein Versuch seinerseits, in unserem Leben sich festzusetzen. Dies ist der Fall, wenn etwa die Annäherung einer Person als ekelhaft empfunden wird [...]. (Kolnai 2007: 61)

Pointiert beschreibt Kolnai an dieser Textstelle den Unterschied zwischen einem Gegenstand, der von Natur aus ekelhaft wirkt und demjenigen, der aus personenbezogenen Gründen zum Ekelhaften denunziert wird. Im Falle der Protagonistin handelt es sich vorwiegend um die oben erwähnten „funktionsfähigen Gegenstände“ und der Ekel resultiert aus ihrer eigenen „Entscheidung“.

Eine Textstelle aus der Erzählung, dient als ein Beispiel für diesen Zustand und akzentuiert, wie divergierend, unterschiedlich und individuell diese „Entscheidungen“ sind.

Er war ein wirklich lieber Kerl. Danach hat mir lange kein Mann mehr so richtig gefallen. Schon nach dem zweiten oder dritten Abend wußte ich, ich würde ihn irgendwann hassen wegen seiner feuchten Aussprache oder wegen der Art, wie er an seinem Schnurrbart zwirbelte [...]. (Dörrie 1990: 113)

Wenn ihr Vorgehen so wie ihre Sensitivität näher betrachtet werden, schließt es sich heraus, dass sie vorweg auf diverse Verhaltensweisen entschieden und konditioniert reagiert. Darüber hinaus gibt es weder Gemeinsamkeiten zwischen diesen Konditionierungen noch eine plausible Erklärung.

Obwohl ihr das Ekelgefühl Grenzen setzt und ihren Alltag und ihre Beziehungen im negativen Sinne beeinflusst, beschreibt sie sich selbst nur als „kritisch“ (ebd.), und sieht ihre Lage als eine vorübergehende Phase. Hiermit werden ihre Gefühle und Erfahrungen, die das Ekelempfinden angehen, reduziert und verdrängt, was daher die Möglichkeit für eine Erkenntnis ausschließt. Sie sieht die Lösung in der Liebe und glaubt, wenn man jemanden wirklich liebt, eine feste Bindung zu ihm pflegt, könnten auch seine Fehler übersehen werden. „Die Theorie des Ekels ist insofern ein Gegenstück - wenn auch kein symmetrisches - zur Theorie der Liebe [...].“ 
(Menninghaus 1999: 7). Die namenlose Protagonistin betrachtet dies genauso und hegt den Glauben, dass Ekel dort nicht existieren kann, wo Liebe vorhanden ist.

Ich habe gedacht, es hört irgendwann auf, es hört auf, wenn ich den Menschen finde, den ich wirklich mag, so ganz und gar mit all seinen Fehlern. Daß mich meine Eltern wahnsinnig gemacht haben, ist doch ganz natürlich, nicht? (Dörrie 1990: 111)

Die mangelnde Liebe gegenüber den Eltern soll als Begründung für ihren Ekel fungieren. Sie nimmt an, dass sich der Ekel von selbst auflöst, sobald sie eine auf Liebe basierende Beziehung eingeht. Ihre Vermutungen erweisen sich jedoch als verfehlt. Im weiteren Handlungsverlauf wird vermittelt, dass in der Gegenwart des Ekels die Liebe zum Scheitern verurteilt ist.

Nach einer Zeit verliebt sich die Protagonistin und heiratet den Mann, von dessen Perfektion sie überzeugt ist. Die Beziehung wirkt eine Zeitlang harmonisch, bis er sich entscheidet, für das Mittagsessen nach Hause zu kommen. Während dieser Mittagszeit beobachtet sie, dass ihr Mann die Gewohnheit hat, unterschiedliche Speisen auf den Teller zu häufen und sie anschließend zu verrühren. Das Argument, ,[...] daß in den meisten Speisen eine Möglichkeit des Ekelhaften steckt [...]“ (Kolnai 2007: 36), wird damit bekräftigt. Somit stellt es sich heraus, dass die Erscheinung und die Mischung sämtlicher Nahrungsmittel miteinander auf sie ebenfalls verstörend wirken und als Ekelauslöser dienen. Der folgenden Textstelle wird besondere Aufmerksamkeit gewidmet, da die Protagonistin hier zwei unterschiedlichen Ekelerfahrungen durch die Kopplung von zwei Sinnesorganen, nämlich des Seh- und dem Gehörsinns, gleichzeitig ausgesetzt ist.

Er hat das ganze Essen auf seinem Teller zu einem Berg zusammengeschoben und zu einem Brei verrührt. Ich habe gemerkt, wie mir ganz plötzlich kalt wurde, eisig kalt [...]. Immer wenn er mit der Gabel in diesen Brei stach, gab es einen schmatzenden Laut [...]. [...] ich bin schnell aufgestanden und ins Bad gelaufen. Ich hatte Angst.

Ich habe es genau vor mir gesehen, wie er die Gabel mit dem Brei in den Mund schiebt, runterschluckt, wie jetzt der Brei in seinem Magen liegt und vor sich hingärt. Es hat mich vor Ekel geschüttelt. (Dörrie 1990: 115)

Es entsteht eine Masse von undefinierbaren „mischmaschartigen“ (Kolnai 2007: 35) und „unpassender Speisemischungen“ (vgl ebd.), begleitet von einem „schmatzenden Laut" (Dörrie 1990: 115). Kolnai betont, dass der Ekel erst empfunden wird, sobald „die betreffende Speise aus irgendeinem [...] Grunde tatsächlich gegessen werden soll“" (Kolnai 2007: 35). Es ist nicht nur die undefinierbare Konsistenz der Speise, die auf sie verstörend wirkt, sondern auch, dass diese Mischung von ihrem Mann gegessen werden soll.

In ihrem Artikel A Perspective on Disgust (1987), beschreiben Paul Rozin (1936) und April E. Fallon (1953) die Abneigung gegen Speisen mit den folgenden Worten: „Distaste is a type of rejection primarily motivated by sensory factors. The focus is on bad taste and/ or smell but may include texture or appearance" (Rozin u.a. 1987: 24). Die Konsistenz ebenso wie die Erscheinung der unpassenden Speisen deuten auch hier auf einen schlechten Geschmack hin. An dieser Stelle wird der Sehsinn in den Mittelpunkt gerückt, ,[...] etwas zu ,,sehen“ heißt in ganz anderem Sinne es zu 
„kennen“ [...]“ (Kolnai 2007: 27). Der Mann hat eine individuelle Art, die Speisen zusammenzustellen. Es sind die Art und Weise, die der Protagonistin nicht vertraut sind, der Sehsinn ist auf etwas gerichtet, das unbekannt, was demzufolge auch zu einem Unbehagen, einer starken Abwehr führt. Außerdem muss sie das Ekelerregende nicht nur sehen, sondern zugleich auch hören. Dieses Zusammenwirken verdoppelt ihr Leiden und ruft bei ihr gemischte Gefühle hervor. Demzufolge kommt neben dem Ekel ein weiterer Gefühlszustand zum Tragen: die Angst.

Kolnai betont, dass „Angst und Ekel [...] „Reaktionen“[...] Antworten auf störende Einwirkungen [...]“" (ebd.: 9) sind. Die körperlichen Symptome, ebenso so wie der Drang, das Umfeld zu wechseln, sind eine Reaktion, eine Antwort auf einen Zustand, der nicht geduldet werden möchte. Es ist ,[...] die Erfahrung einer Nähe, die nicht gewollt wird“( Menninghaus 1999: 7). Des Weiteren sieht Kolnai in der Angst ein „Schutzbedürfnis“ (Kolnai 2007: 14) vor einer gewissen „Bedrohung, der Gefahr“ (vgl. ebd.). Die Angst resultiert aus der Besorgnis, sich nicht beherrschen und die Kontrolle über das eigene Handeln verlieren zu können. Um diese Gefahr zu vermeiden, setzt sie wieder einen Schutzmechanismus in Kraft und entfernt sich aus der Nähe des Ekelauslösers.

Mit ihrer Einbildungskraft stellt sich die Protagonistin vor, was mit der Nahrungsaufnahme im Körper ihres Mannes geschieht, wie die Nahrung sich in einen feuchten Speisebrei verwandelt und im Magen verdaut wird. Diese Textstelle ist eine Anspielung auf die Erzählung Liebe und dennoch (1987) von Alfred Polgar (18731955), in welcher ein Mann die Nahrungsaufnahme seiner Frau genauso plastisch und detailliert schildert. Obwohl die Protagonistin intendiert, dem steigernden Ekelgefühl in etlichen Wegen zu entfliehen, wird der Ekel durch ihre Einbildungskraft gefüttert und lässt sie nicht los.

In ihrer Vorstellung gibt es Vorschriften, gelernte Assoziationen, die die Nahrungsaufnahme und die Zubereitung der Speisen betreffen und die einzuhalten sind. Das Zusammenmischen der Speisen durch ihren Ehemann versucht sie zu verhindern, indem sie Gerichte wie Suppe, Fleisch und Salat vorbereitet, die keine breiigen Eigenschaften haben und nicht miteinander vermischt werden können. Unzufrieden mit dem Kochverhalten seiner Frau entscheidet sich der Mann anschließend, für sich selbst zu kochen. Die Küche als ,[...] Produktionsort einer jeweils spezifischen Form von Weiblichkeit“ (Miklautz u.a. 1999: 12) verwandelt sich in den Produktionsort ihres Mannes. Mit seinem Eingriff in die Küche markiert der Ehemann das Ende ihrer Herrschaft in diesem Raum. In ihrem Werk Essen und Psyche beschreibt Gisla Gniech (1937-2015), die gewichtige Rolle der Frau in der Küche und betont, dass, ,[d]ie Hausfrau entscheidet, welche Speise auf den Tisch kommt" (Gniech 1995: 179). Der aufgezwungene Verzicht auf die Rolle in der Küche, diese Entmachtung führt die Protagonistin in eine tiefe Isolation und die Beziehung verliert folglich ihr Gleichgewicht.

In einer weiteren Textstelle erzählt sie über einen verstörenden Albtraum und dessen Auswirkungen auf ihre Beziehung zu ihrem Mann. 
Einmal habe ich geträumt, ich läge neben ihm im Bett, und plötzlich habe ich etwas Warmes, Feuchtes auf meiner Haut gespürt, und als ich mich umgedreht habe, habe ich gesehen, wie sein Bauch aufgeplatzt war und ein dicker, gelblichgrüner Brei aus ihm herausfloß, immer mehr wurde, über die Bettdecke auf den Boden rann, das Zimmer füllte, aus den Fenstern quoll, immer höher stieg und drohte mich zu ersticken. Ich muß vor Angst geschrien haben. Als ich erwachte, hielt er mich im Arm. Seine Berührung war schlimmer als der Traum. Von da an schliefen wir getrennt. (Dörrie 1990: 117)

Doris Dörrie verwendet an dieser Textstelle mehrere Ekelmotive zugleich. Die Quelle dieser Motive ist der „menschliche Leib“ (Kolnai 2007: 37). Der aufgeplatzte, versehrte und von jeder Vollkommenheit entfernte, kontaminierte Körper des Mannes im Traum löst bei der Protagonistin heftige Angst aus. Mit seinen „Körperöffnungen“ (Menninghaus 1999: 16) und „Körperflüssigkeiten“ (vgl. ebd.) wird der Mann selbst zum Gegenstand des Ekels und verkörpert das Ekelhafte. Der Tod erscheint hier in grellster Anschaulichkeit, dehnt sich in der Form einer flüssigen, klebrigen Masse aus und die Protagonistin fühlt sich dadurch davon bedroht. In ihrem Traum sieht sie einen „Übergang des Lebendigen in den Zustand des Toten“ (Kolnai 2007: 29). Es ist hier weder der Tod selbst noch der Tod des Mannes, der bei der Protagonistin zu einem wahrhaftigen Schrecken und zu Ekel führt. Kolnai akzentuiert ebenfalls, „daß niemals das Tote als bloßes nichtfunktionierendes Lebendiges ekelhaft ist"“ (vgl. ebd.). Hier ist es der Anblick des graduellen Wandels im Körper des Mannes, nach dem Tod, der bei ihr zu einem Entsetzen führt. Die Berührung des Mannes, der sie zu beruhigen versucht, ist unerwünscht, da die Protagonistin zwischen dem ekelerregenden Körper im Traum, der nun abgelehnt wird, und ihrem Mann nicht mehr unterscheidet. Sie empfindet seine Berührung als Kontamination. Der Traum löst eine Abneigung aus und führt zu einer tiefgreifenden Veränderung in ihrer Beziehung. Des Weiteren resümiert er nicht nur den Zustand einer problematischen Figur, die von Ekel befangen ist, er illustriert ebenfalls jenen Punkt, an welchem ihre Beziehung angelangt ist. Der deformierte Körper symbolisiert den drastischen Wandel in ihrem Leben und ihrer zerstörten Beziehung.

Nach diesem verstörenden Traum zieht sich die Protagonistin in ihre Innenwelt zurück und bricht den Kontakt zu ihrem Mann vollends ab. Der Mann hingegen möchte ihre Aufmerksamkeit und sucht Kontakt zu ihr.

\footnotetext{
Er schloß die Tür ab und sagte, er müsse mit mir reden. [...] Er nahm mich am Arm und zwang mich, mich hinzusetzen. [...] Dann nahm er die Gabel. Kartoffelbrei, Spinat und Spiegeleier. An mehr kann ich mich nicht mehr erinnern. Sie haben mir vor Gericht ein langes Messer gezeigt in einer Plastiktüte. (Dörrie 1990: 117)
}

Der Mord an ihrem Mann ist eine massive Handlung und kennzeichnet eine Schwelle, die sie niemals hatte überschreiten wollen. Ihre Emotionen erreichen somit einen Höhepunkt, da sie zu mehreren Ekelauslösern, wie die Berührung des Mannes, dem Anblick unpassender Speisen zugleich ausgesetzt ist. Sie befindet sich in einer Lage, in welcher ihre Gedanken keinen Einfluss mehr auf ihr Handeln haben.

Die Türe, die von dem Ehemann von innen geschlossen wird, schließt für sie endgültig den Ausweg, sich aus dem Umfeld $\mathrm{zu}$ distanzieren. Durch die ,sich aufzwingende Nähe“" (vgl. Kolnai 2007: 23) des Ehemannes eskaliert die Situation und 
bildet den Anlass für den Mord. Dies erweist sich als Folge der seit Jahren angesammelten unangenehmen Erfahrungen.

Die Aussage „Disgust can kill love and friendship [...]” (Miller 1997: 24),findet ihre Bestätigung in der vorgeführten Beziehung der Protagonistin. Ihre Störung, die sie nicht verarbeiten kann, löst sie aus allen familiären Beziehungen heraus. Dass sie sich an den Mordfall nicht erinnern kann, zeigt nicht nur den Grad ihrer Störung, sondern auch wie dominant die Emotionen Angst und Ekel in ihrem Leben sind.

Das Ekelmotiv wird in der Literatur so wie im Film sehr häufig verwendet. Doch nur in geringem Ausmaß wird es als Leitmotiv in den Fokus gerückt. Abstoßende Bilder und Inszenierungen in Slasher Filmen oder sämtliche Textstellen in literarischen Werken, wie zum Beispiel in Jerzy Kosinskis‘ (1933-1991) Werk Der bemalte Vogel (1965), können zu einem Unbehagen führen. Obwohl der Ekel in diesen Beispielen vorhanden ist, dient er keineswegs als Leitmotiv. Dörrie befasst sich in ihrer Erzählung nicht nur mit der psychologischen aber auch mit der ästhetischen Dimension des Ekels.

Dörries Erzählung gehört zu den wenigen literarischen Texten, die sich hauptsächlich mit der Ekelthematik auseinandersetzen: „Almost all objects that qualify as digusting by our criteria are animals or parts of animals, animal body products, [...]" (Rozin 1987: 27). Doch die Autorin denkt hier außerhalb des üblichen Rahmens, breitet diese Liste der Ekelauslöser aus und bezieht sich nicht allein auf Tiere und Tierprodukte. Die hohe Sensitivität der Protagonistin gegen viele unterschiedliche Gegenstände machen aus ihr eine zugespitzte, unberechenbare Person. Die Irrationalität so wie die Instabilität der Hauptfigur werden durch ihre Ekelempfindung betont. An einer kurzen Textstelle gelingt es der Autorin, den Ekel- als einer der Basisemotionen in seiner Vielfältigkeit zu veranschaulichen.

\section{Literaturverzeichnis}

Darwin, Charles (1872): The Expression of the Emotions in Man and Animals. London: John Murray.

Dörrie, Doris (1990): Mit Messer und Gabel. In: Dörrie, Doris: Was wollen Sie von mir? Zürich: Diogenes, 109-117.

Gniech, Gisla (1995): Essen und Psyche. Über Hunger und Sattheit, Genuß und Kultur. Berlin, Heidelberg, New York: Springer.

Kolnai, Aurel (2007): Ekel, Hochmut, Haß. Zur Phänomenologie feindlicher Gefühle. Frankfurt am Main: Suhrkamp.

Menninghaus, Winfried (1999): Ekel. Theorie und Geschichte einer starken Empfindung. Frankfurt am Main: Suhrkamp.

Miglautz, Elfie / Lachmayer, Herbert / Eisendle, Reinhard (1999): Einleitung. Die Küche. Zur Geschichte eines architektonischen, sozialen und imaginativen Raums. In: Miglautz, Elfie / Lachmayer, Herbert / Eisendle, Reinhard (Hg.): Die Küche. Zur Geschichte eines architektonischen, sozialen und imaginativen Raums. Wien/ Köln/ Weimar: Böhlau, 9-16.

Miller, Ian William (1997): The Anatomy of Disgust. Cambridge/ London: Harvard University Press. 